\title{
A novel endoscopic technique for closure of a large esophageal perforation using the clip-and-snare method with the prelooping technique
}

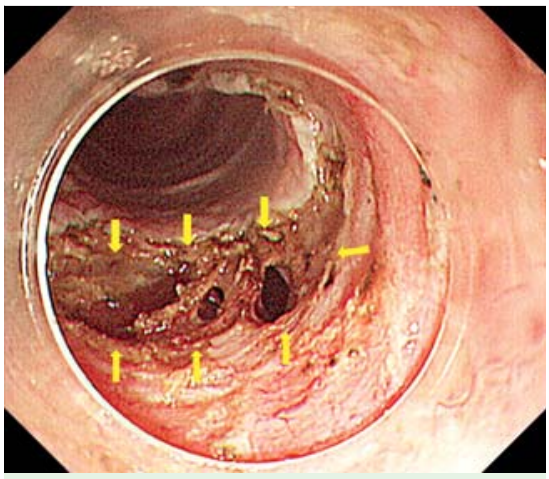

Fig. 1 Endoscopic view showing a large perforation (yellow arrowheads) in the cervical esophagus during endoscopic submucosal dissection.

It is sometimes difficult to close a large perforation using endoclips and over-thescope clips, and surgery may be required [1]. We report a novel endoscopic technique for closure of a large perforation using the clip-and-snare method with the prelooping technique.

A 76-year-old man with a metachronous esophageal cancer, which developed at a scar in the cervical esophagus that resulted from a previous endoscopic submucosal dissection (ESD), underwent a second ESD. A large perforation occurred during submucosal dissection because of severe fibrosis associated with the previous ESD ( $\bullet$ Fig. 1). Closure of the perforation could not be achieved by endoscopic clip placement because of its large size. Therefore, we endoscopically closed the perforation using an endoclip with the clip-and-snare method and prelooping technique, as has been applied in the traction method for ESD $[2,3]$.

First, a snare (SD-210L-15; Olympus, Tokyo, Japan) was prelooped around the transparent hood (D-201-11804; Olympus) attached to a single-channel upper gastrointestinal endoscope (GIF-Q260J; Olympus) ( $\bullet$ Fig. 2a), which was then advanced towards the mucosal defect. The anal side of the mucosal perforation was grasped using an endoclip (HX-610-090; Olympus) passed through the scope channel and was not released. Subsequently, the prelooped snare was loosened from the transparent hood ( $\bullet$ Fig. 2 b), and the
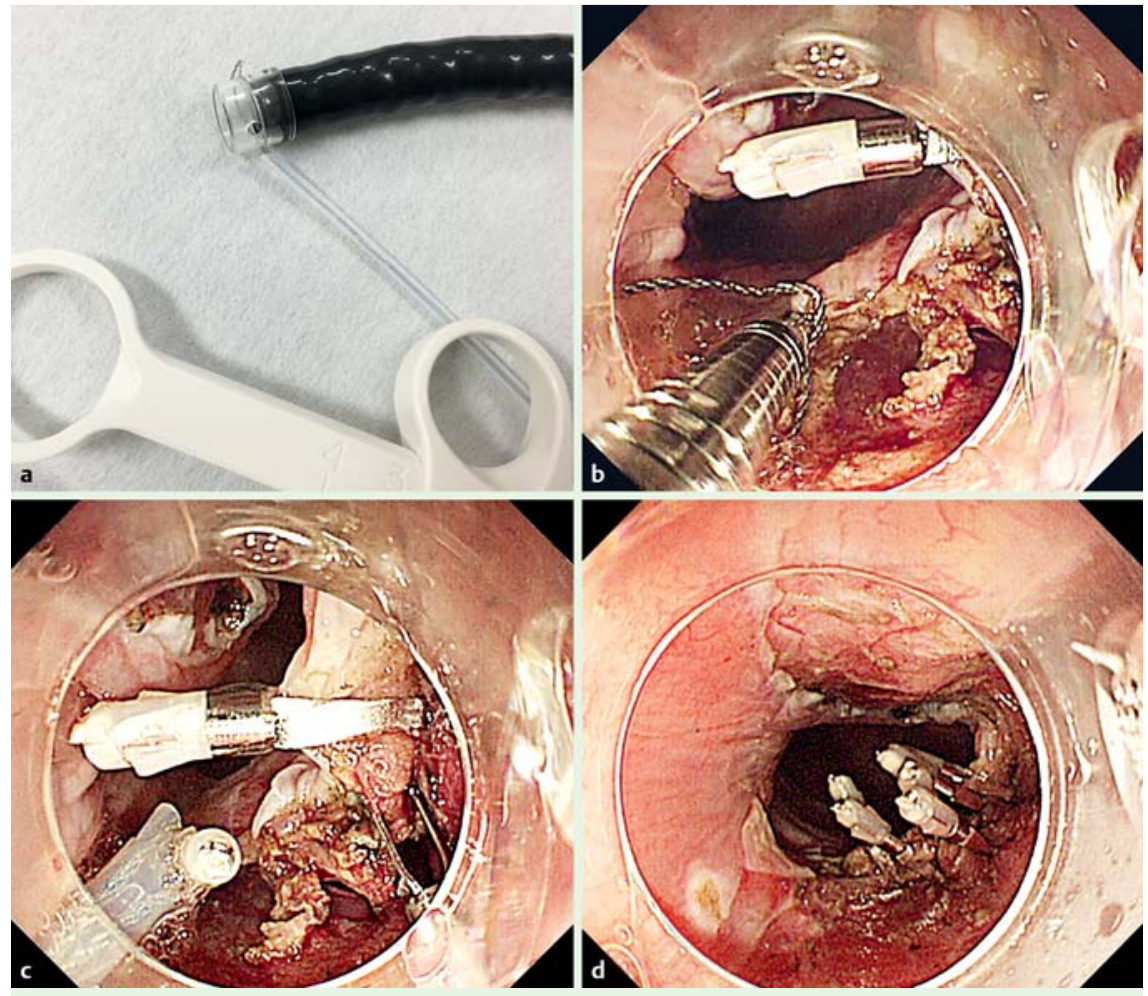

Fig. 2 The clip-and-snare method with prelooping technique. a A snare was prelooped around the transparent hood attached to a single-channel upper gastrointestinal endoscope. b The prelooped snare was loosened from the transparent hood. c The endoclip that had previously been used to grasp the anal side of the mucosal perforation was grasped with the snare. $\mathbf{d}$ Endoscopic view showing successful closure of the perforation.

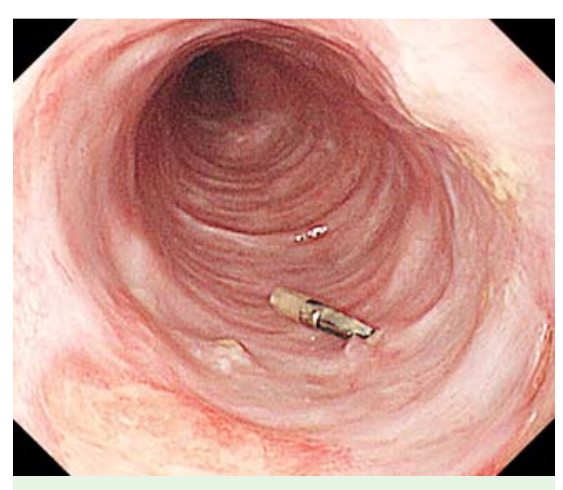

Fig. 3 Endoscopic view 2 months later showing an ulcer scar.

endoclip was grasped with the snare and released from the forceps ( $\bullet$ Fig. 2 c). The snare was pulled from the mouth, resulting in narrowing of the perforation, which could then be closed using endoclips ( Fig. 2d; $\odot$ Video 1 ).

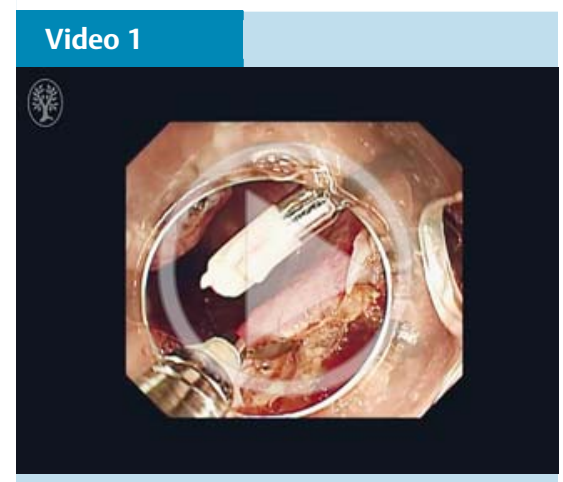

A large esophageal perforation is closed using the clip-and-snare method with prelooping technique.

Following this procedure, the patient developed no symptoms, except for a slight fever and elevation of the C-reactive protein (CRP) level. An ulcer scar was observed 2 months post-ESD ( $\bullet$ Fig.3). 
This novel technique involving a device that is routinely used for endoscopic therapy may be a helpful and easy procedure for closure of a large perforation in the digestive tract.

Endoscopy_UCTN_Code_TTT_1AO_2AI

Competing interests: None

Yasuaki Nagami, Masatsugu Shiba, Masaki Ominami, Kazunari Tominaga, Toshio Watanabe, Yasuhiro Fujiwara, Tetsuo Arakawa

Department of Gastroenterology, Osaka City University Graduate School of Medicine, Osaka, Japan

\section{References}

1 Verlaan T, Voermans RP, van Berge Henegouwen $M I$ et al. Endoscopic closure of acute perforations of the GI tract: a systematic review of the literature. Gastrointest Endosc 2015; 82: 618-628 e615

2 Yamada S, Doyama $H$, Ota R et al. Impact of the clip and snare method using the prelooping technique for colorectal endoscopic submucosal dissection. Endoscopy 2016; 48: $281-285$

3 Yoshida N, Doyama H, Ota R et al. The clipand-snare method with a pre-looping technique during gastric endoscopic submucosal dissection. Endoscopy 2014; 46 (Suppl. 01): E611-E612
Bibliography

Dol http://dx.doi.org/

10.1055/s-0042-110488

Endoscopy 2016; 48: E250-E251

(c) Georg Thieme Verlag KG

Stuttgart · New York

ISSN 0013-726X

\section{Corresponding author}

\section{Kazunari Tominaga, MD}

Department of Gastroenterology

Osaka City University Graduate School of Medicine 1-4-3, Asahimachi, Abeno-ku

Osaka, 545-8585

Japan

Fax: +81-6-6645-3813

tomy@med.osaka-cu.ac.jp 